Reis et al., Revista Brasileira de Higiene e Sanidade Animal (v.14, n. 2) p. 269 - 280 abr - jun (2020)

Revista Brasileira de Higiene e Sanidade Animal

Brazilian Journal of Hygiene and Animal Sanity

ISSN: 1981-2965

\title{
Plantas tóxicas para animais produção da região Sudoeste da Bahia. Uma Revisão
}

Toxic plants for livestock in the Southwest region of Bahia, Brazil. A review

\section{Suélen Dias Silva dos Reis ${ }^{1}$, Marilúcia Campos dos Santos ${ }^{1}$, Juliana Targino Silva Almeida e Macêdo ${ }^{2}$, Pedro Miguel Ocampos Pedroso ${ }^{2} *$}

Resumo: Foi realizado um levantamento sobre as principais plantas tóxicas para animais de produção na região Sudoeste do estado da Bahia, Brasil. Foram entrevistados 120 pessoas, incluindo produtores rurais, médicos veterinários e técnicos agrícolas de 30 municípios. No total, 80 surtos de intoxicação por plantas tóxicas foram registrados. As espécies de animais acometidas foram: bovina, caprina, ovina e equina. As principais espécies de plantas tóxicas, em ordem decrescente de surtos foram, Pteridium arachnoideum, Amorimia rigida, Ricinus communis e Froelichia humboldtiana. Outras plantas apontadas como causadoras de intoxicação também foram apontadas, Brachiaria spp., Marsdenia spp., Manihot spp., Mimosa tenuiflora, Thiloa glaucocarpa, Enterolobium contortisiliquum, casca de Coffea arabica e Asclepias curassavica. As espécies Imperata brasiliensis Trin., Senna acurensis, Capparis jacobinae e Cecropia hololeuca Miq. precisam ter a toxicidade confirmada. Novos estudos para comprovar a toxicidade das plantas mencionadas devem ser executados, a fim de elucidar as informações sobre intoxicação por plantas. Com base nos resultados, conclui-se que as intoxicações por plantas tóxicas são frequentes em ruminantes e equinos na região Sudoeste da Bahia e representam importante causa de perdas econômicas para os produtores rurais.

Palavras chave: intoxicação; Pteridium arachnoideum; ruminantes; equídeo; nordeste do Brasil.

Abstract: A survey on toxic plants for livestock was performed in the Southwest region of the state of Bahia, Brazil. One hundred twenty people were interviewed, including farmers, veterinarians, and agrarian technicians from 30 municipalities. In total 80 outbreaks of intoxication by toxic plants were recorded. The affected animal species were the bovine, caprine, ovine and equine. The main species of toxic plants, in decreasing order of outbreaks were Pteridium arachnoideum, Amorimia rigida, Ricinus communis and Froelichia humboldtiana. Other plants pointed as causing intoxication were Brachiaria spp., Marsdenia spp., Manihot spp., Mimosa tenuiflora, Thiloa glaucocarpa, Enterolobium contortisiliquum, skin of Coffea arabica and Asclepias curassavica. The species Imperata brasiliensis Trin., Senna acurensis, Capparis jacobinae and Cecropia hololeuca Miq. need to have confirmed toxicity. New studies testing the toxicity of the mentioned plants must be performed, in order to widen the information about poisoning by plants. Based in the results, it is concluded that poisoning by toxic plants are frequent in livestock in the Southwest region of the state of Bahia and represent an important cause of economic losses to farmers.

Keywords: poisoning; plants; ruminant; equidae; Bahia, northeastern Brazil.

Autor para correspondência: E-mail: * pedrosovet@yahoo.com.br 
Recebido em 10.01.2020. Aceito em 30.06.2020 http://dx.doi.org/10.5935/1981-2965.20200024

1 Setor de Patologia Veterinária, Universidade Federal do Recôncavo da Bahia (UFRB), Cruz das Almas, BA 44380-000. suelen.estrela@hotmail.com, marilucampos@gmail.com

2 Laboratório de Patologia Veterinária, Fundação Universidade de Brasília (UnB), Campus Universitário Darcy Ribeiro, Via L4 Norte s\n, Brasília, DF 70910-970.

pedrosovet@yahoo.com.br, ,jtsam_targino@yahoo.com.br

\section{Introdução}

No Brasil, atualmente são conhecidas cerca de 130 plantas tóxicas para ruminantes e equídeos (TOKARNIA et al., 2012; PESSOA et al., 2013). Apesar do extenso estudo e da vasta literatura relacionada às plantas tóxicas no país, ainda há uma carência de informações em relação à frequência das intoxicações por plantas em determinadas regiões do Brasil (TOKARNIA et al., 2012).

O estado da Bahia destaca-se pelos elevados números de animais de produção, com uma população de 10.828.409 bovinos, 2.458.179 de caprinos, 2.926.601 de ovinos e 485.356 equinos (IBGE, 2014). Informações sobre plantas tóxicas e sobre perdas que estas ocasionam no cenário econômico rural da Bahia são escassas. Já em outros estados do Nordeste do Brasil, cresce o número de pesquisas nessa área (SILVA et al., 2006; PIMENTEL et al., 2007; ASSIS et al., 2009; MELLO et al., 2010; BEZERRA et al., 2012). O estudo das intoxicações por plantas tem avançado no Brasil e demonstrado a elevação do percentual das mesmas dentre as causas de morte dos animais (TOKARNIA et al., 2012; PESSOA et al., 2013). Neste contexto, o presente trabalho teve como objetivo realizar um levantamento de surtos ocasionados por plantas tóxicas para animais de produção da região Sudoeste da Bahia.

\section{Material e Métodos}

O levantamento foi realizado aleatoriamente na zona rural de 30 municípios dos 39 que compõem a região Sudoeste da Bahia. As visitas foram realizadas no período de março a novembro de 2014. Os municípios visitados foram: Anagé, Barra do Choça, Boa Nova, Cândido Sales, Cravolândia, Encruzilhada, Firmino Alves, Ibicuí, Irajuba, Itambé, Itapetinga, Itaquara, Itarantim, Itiruçu, Itororó, Jaguaquara, Jequié, Lafaiete Coutinho, Lagedo do Tabocal, Macarani, Maiquinique, Manoel Vitorino, Maracás, Planaltino, Planalto, Poções, Ribeirão do Largo, Santa Inês, Tremedal e Vitória da Conquista. A região e os municípios estudados estão apresentados na Figura 1.

]Foram entrevistados produtores rurais, médicos veterinários, agrônomos e técnicos da área. O questionário aplicado aos entrevistados era composto por três formulários, semelhantes aos empregados por SILVA et al., (2006). O primeiro formulário (F1) continha uma relação de 
42 plantas de potencial comprovadamente tóxico (RIET-CORREA et al., 2011; TOKARNIA et al., 2012). O segundo formulário (F2) era destinado para registrar plantas mencionadas pelos entrevistados e que não foram listadas no formulário 1 . No terceiro formulário $(\mathrm{F} 3)$ foi específico para os relatos de intoxicação. Para facilitar a identificação das plantas, foi utilizado o livro Plantas Tóxicas do Nordeste (RIETCORREA et al., 2011) e Plantas Tóxicas do Brasil (TOKARNIA et al., 2012). Os sinais clínicos mencionados pelos entrevistados foram interpretados pela equipe e descritos nos formulários.

Amostras de plantas do F2 foram devidamente coletadas (ROTTA et al., 2008) e encaminhadas para identificação botânica na Universidade Estadual de Feira de Santana. Dados do F3 foram compilados e arquivados em livros de registro do Setor de Patologia Veterinária da Universidade Federal do Recôncavo da Bahia (SPV-UFRB).

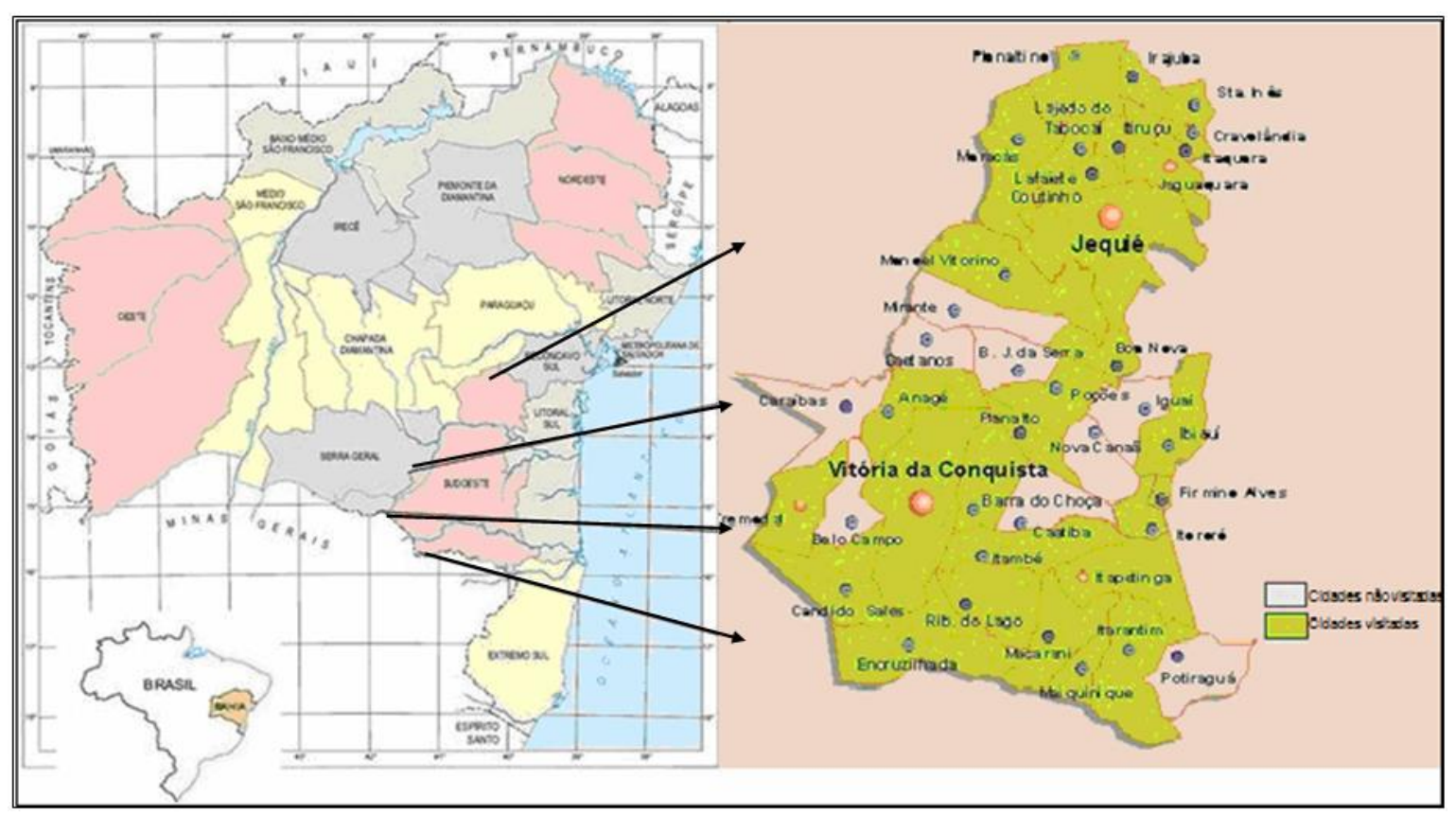

Figura 1: Mapa do estado da Bahia com destaque para a região Sudoeste. Na cor verde, municípios onde foram realizadas as entrevistas. Fonte: Adaptado de SEIBA, 2003.

\section{Resultados e Discussão}

Foram realizadas 120 entrevistas, com uma média de quatro entrevistados por município, destas, houve relato de 75 surtos de intoxicação por plantas comprovadamente tóxicas de interesse pecuário e 5 surtos por Asclepias curassavica considerada de pouco interesse pecuário, além do relato de 4 plantas mencionadas como tóxicas pelos entrevistados da região, mas cuja toxicidade não foi comprovada. 
Os resultados obtidos através da aplicação dos formulários 1 e 2 aos entrevistados são mencionados a seguir. Plantas de toxicidade comprovada:

\section{Plantas de ação radiomimética}

\section{Pteridium}

arachnoideum (samambaia) - Vinte e cinco surtos foram relatados em bovinos (corte e leite), que totalizaram 230 acometidos e 144 mortes. $\mathrm{Na}$ região Sudoeste da Bahia, $P$. arachnoideum é conhecida popularmente como feto (Figura 2A). Os casos ocorreram em qualquer mês do ano. Os sinais clínicos relatados, foram hematúria, apatia, anorexia, perda de peso seguidos de morte. Um entrevistado do município de Cravolândia relatou um surto em que os bovinos apresentaram diversas hemorragias seguidas de morte (intoxicação aguda), e outros dois relataram que alguns animais apresentam roncos e tosses. O curso clínico variou de cinco dias a três meses. Animais adultos foram os mais afetados (até quatro anos).

Plantas que causam falha cardíaca aguda associada ao exercício

Amorimia rigida (Mascagnia rigida) (tinguí) - Foram descritos dezoito surtos em bovinos após período seco e associados ao exercício. Aproximadamente 207 animais morreram. Os sinais clínicos foram sialorreia, tremores musculares, dificuldade para manter em estação, seguido de morte.

\section{Plantas que causam sintomatologia nervosa}

Ricinus communis (mamona) Foram descritos oito surtos em bovinos, com 21 mortes. Os sinais clínicos foram andar cambaleante, queda, decúbito lateral e morte. Em um dos surtos, de um rebanho de 51 bovinos de corte, 20 morreram subitamente após serem introduzidos em pasto com a planta.

Marsdenia spp. (mata calado, rabode-tatu) - Quatro surtos foram relatados em bovinos. Em três surtos os animais caíam ao se movimentar. Adicionalmente outros sinais clínicos relatados foram tremores generalizados, queda, decúbito e morte.

Coffea arabica L. (casca do fruto do café) - Um médico veterinário relatou que em 2011 dois equinos se intoxicaram por casca de café (Figura 2B) durante uma exposição agropecuária no município de Vitória da Conquista. A casca de $C$. arabica era utilizada como cama de baia. Os sinais clínicos relatados foram incoordenação motora, depressão e morte.

\section{Plantas cianogênicas}

Manihot spp. (maniçoba) - Três surtos foram descritos em bovinos. Em um surto os animais ingeriram tanto os tubérculos quanto as partes aéreas da planta. Os sinais clínicos caracterizaram-se por sialorreia, andar cambaleante, dificuldade para se manter em estação, timpanismo, decúbito e morte. 
Plantas que causam malformações

Mimosa tenuiflora (jurema preta) Dois surtos ocorreram em caprinos e ovinos. Na primeira espécie ocorreram casos de aborto e malformações no município de Cravolândia. No município de Santa Inês, duas ovelhas consumiram a planta durante a gestação, sendo que uma teve parto gemelar, onde as crias apresentaram membros torácicos malformados, dificuldade para se alimentar e morte após dois dias. A segunda ovelha abortou.

\section{Plantas nefrotóxicas}

Thiloa glaucocarpa (vaqueta) Dois surtos foram descritos em bovinos. A intoxicação ocorre principalmente no início do período chuvoso. Aproximadamente um mês após o início das chuvas, os animais apresentam anorexia, emagrecimento e edema ventral da região abdominal e em membros pélvicos, vindo a óbito.

\section{Plantas que causam fotossensibilização primária}

Froelichia humboldtiana (ervanço)

- Foram descritos seis surtos em equinos, com 20 animais acometidos e sem morte. Os sinais clínicos relatados foram perda de pelo e úlceras crostosas nas áreas brancas e/ou despigmentadas do corpo. Quatro entrevistados afirmaram que os surtos acontecem há mais de dez anos, anualmente, na época das chuvas (abril a junho), com duração de cinco meses, sem predisposição por idade e a recuperação é obtida após troca de pastagem e aplicação de cicatrizante.

\section{Plantas que causam fotossensibilização secundária}

Brachiaria spp. (capim braquiária)

- Foram descritos cinco surtos, sendo dois em ovinos e três em bovinos. Na região há principalmente $B$. decumbens e $B$. brizantha. Os ovinos apresentaram apatia, lesões de pele e edema nas orelhas, após permanecerem aproximadamente por 30 dias em pasto contendo capim braquiária. Nos bovinos os sinais clínicos foram alopecia, escleras ictéricas e apatia.

Enterolobium contortisiliquum (tamboril, timbaúva) - Um surto ocorreu em bovinos, com quatro animais intoxicados. Houve lesões de fotossensibilização, mas o entrevistado não soube informar sobre a duração da doença. $\underline{\text { Plantas experimentalmente tóxicas para }}$ bovinos sem interesse pecuário:

Asclepias curassavica (oficial-desala, erva-de-rato) - Cinco surtos foram relatados, sendo três em bovinos e dois em equinos. Em bovinos os sinais clínicos se caracterizaram por apatia, anorexia, dispneia, decúbito e morte em até três dias. No município de Itaquara, bovinos se intoxicaram na época da seca; os animais apresentaram tremores musculares, eructações e sialorreia. 
Reis et al., Revista Brasileira de Higiene e Sanidade Animal (v.14, n. 2) p. 269 - 280 abr - jun (2020)

$\underline{\text { Plantas mencionadas como tóxicas pelos }}$ entrevistados da região, mas cuja toxicidade não está comprovada:

Imperata brasiliensis Trin. (capim Sapé) - Dois entrevistados, um de Jaguaquara e um de Lafaiete Coutinho relataram que a planta promove em bovinos um quadro de hematúria, perda de peso e óbito e que a ocorrência é anual.

Senna acuruensis (canjoão) - Um entrevistado de Cravolândia relatou que os bovinos apresentam um quadro de diarreia enegrecida. A planta é arbustiva e as vagens seriam as partes tóxicas.

Capparis jacobinae (icó) - Um entrevistado do município de Santa Inês relatou que um ovino se intoxicou por $C$. jacobinae (Figura 2C), apresentando alopecia generalizada, especialmente na cauda.

Cecropia hololeuca Miq. (umbaúba) - Um entrevistado de Jaguaquara relatou que em bovinos a ingestão desta planta causa hematúria. $\mathrm{O}$ entrevistado não soube informar sobre a duração da doença.

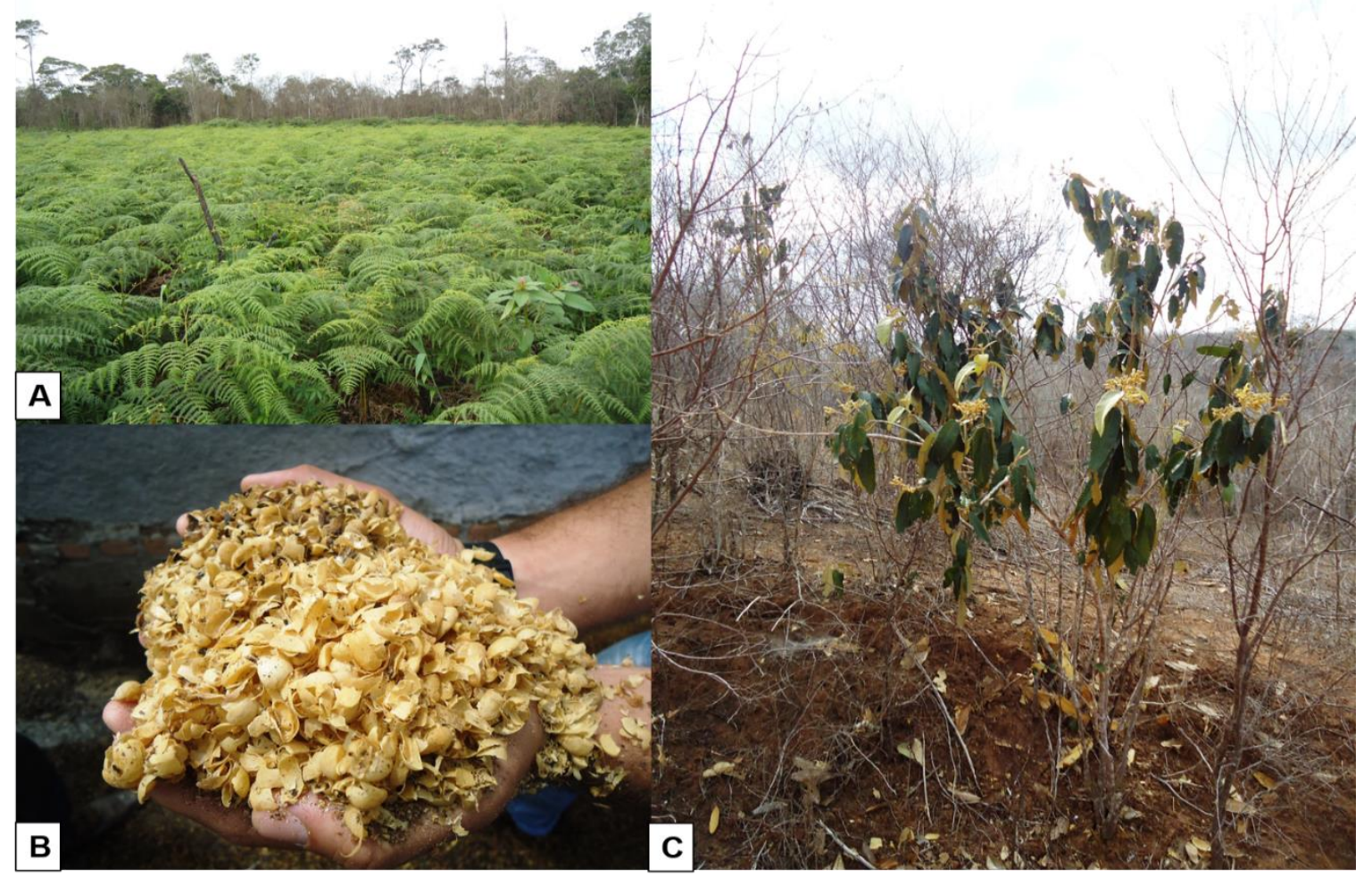

Figura 2: Plantas tóxicas para animais de produção na região Sudoeste da Bahia. (A) Pastagem intensamente invadida por Pteridium arachnoideum em propriedade rural do município de Encruzilhada, BA. (B) Casca de Coffea arabica L. utilizada como cama de baia em exposição agropecuária. (C) Exemplar da planta Capparis jacobinae.

Os resultados do presente trabalho demonstraram a ocorrência de 12 diferentes plantas comprovadamente tóxicas causando surtos e mortalidade em ruminantes e equinos na região Sudoeste da Bahia. 
A planta responsável pelo maior número de surtos na região Sudoeste da Bahia foi Pteridium arachnoideum.

Dos 80 surtos relatados, 25 $(31,25 \%)$ foram causados por $P$. arachnoideum. Diferente de outros inquéritos epidemiológicos sobre plantas tóxicas realizados em outras regiões do Nordeste do Brasil, na qual a principal planta tóxica foi Ipomoea asarifolia (SILVA et al., 2006; ASSIS et al., 2009; MELLO et al., 2010; BEZERRA et al., 2012). P. arachnoideum está presente desde o sul da Bahia até o Rio Grande do Sul, em áreas preferencialmente montanhosas, frias e de boa pluviosidade (TOKARNIA et al., 2012).

Estas condições estão presentes na região estudada e muitas áreas estão extensamente invadidas por esta planta. As formas crônicas da intoxicação são hematúria enzoótica bovina (HEB) e carcinoma de células escamosas no trato alimentar superior e a forma aguda caracterizada pela síndrome hemorrágica (SOUTO et al., 2006; ANJOS et al., 2008; TOKARNIA et al., 2012). HEB caracteriza-se por sinais inespecíficos como hematúria, anorexia, fraqueza, dor abdominal e prostração (GABRIEL et al., 2009), compatíveis com os relatos apresentados. O diagnóstico de HEB é frequente nas regiões Sudeste e Sul do Brasil (FALBO et al., 2005; RISSI et al.,
2007; GABRIEL et al., 2009; SILVA et al., 2009) e mais recentemente no CentroOeste (FURLAN et al., 2014a; FURLAN et al., 2014b). A planta é importante no estado da Bahia apresentando registros de hematúria enzoótica na região sudoeste (REIS et al., 2016a).

A segunda planta mais mencionada nas entrevistas foi a Amorimia rigida. A intoxicação por A. rigida apresentou importância para a espécie bovina. Os sinais clínicos mencionados foram sialorreia e tremores seguidos de morte. A intoxicação ocorre pela presença do ácido monofluoracético que inibe a formação de ATP a nível do ciclo de Krebs, levando os animais a morte súbita associada ao exercício (RIET-CORREA et al., 2011). Caprinos e ovinos também são sensíveis, no entanto não houve relato nestas espécies, possivelmente pelo fato da ovinocaprinocultura não serem muito exercidas na região.

Surtos de intoxicação por Froelichia humboldtiana foram bem característicos. A espécie equina foi a única citada, entretanto na literatura há confirmação de casos em ovinos, bovinos, asininos e muares (PIMENTEL et al., 2007; SOUZA et al., 2007; KNUPP et al., 2014). As áreas despigmentadas da pele são afetadas quando os animais ingerem grandes quantidades da planta (MEDEIROS et al., 2014). 
A intoxicação ocorre principalmente na época das chuvas (RIET-CORREA et al., 2011; KNUPP et al., 2014). Neste estudo a ocorrência foi mencionada principalmente no período chuvoso (abril a junho).

$\mathrm{Na}$ intoxicação por Ricinus communis os animais apresentaram sinais clínicos neurológicos seguido de óbito. Em 2009, foi realizado um levantamento no sertão paraibano, onde $R$. communis foi mencionada causando distúrbios digestivos (ASSIS et al., 2009). A ingestão das folhas e do pericarpo ocasiona sinais clínicos neurológicos, enquanto a ingestão das sementes leva ao quadro de alterações digestivas (VASCONCELOS et al., 2008).

Poucos entrevistados informaram sobre a intoxicação por Mimosa tenuiflora, porém são frequentes casos de abortos e de malformações em caprinos. Os tipos de malformações induzidos são variados, bem conhecidos no semiárido nordestino como queilosquise, artrogripose, micrognatia, hipoplasia ou aplasia de ossos incisivos (PIMENTEL et al., 2007; DANTAS et al., 2010), além de causar aborto e perda embrionária (SANTOS et al., 2012).

Ultimamente têm-se registrado no município de Uauá, sertão (clima semiárido) da Bahia vários surtos de malformações e perdas reprodutivas em ovinos e caprinos onde Poincianella pyramidalis (Tul.) L.P. Queiroz
(=Caesalpinia pyramidalis Tul.) planta popularmente conhecida como "catingueira", "pau-de-rato" e "catinga-deporco" era a planta predominante. Posteriormente foi comprovado experimentalmente em caprinos o efeito teratogênico de Poincianella pyramidalis como causa de perdas embrionárias, aborto e malformações (REIS et al., 2016b).

Thiloa glaucocarpa foi responsável por causar intoxicações no início do período chuvoso, com quadro clínico característico de edema, especialmente nos membros pélvicos. As mesmas observações foram registradas no Piauí (MELLO et al., 2010).

Brachiaria spp. é bem conhecida na região e causa surtos de fotossensibilização hepatógena em bovinos e ovinos. No Brasil, casos de intoxicação por capim braquiária nestas duas espécies são frequentes (TOKARNIA et al., 2012).

Nesse estado já foi registrado um surto importante de intoxicação por Brachiaria decumbens em ovinos (OLIVEIRA et al., 2012).

Outras plantas tóxicas mencionadas, aparentemente de menor importância foram Marsdenia spp., casca do fruto de Coffea arabica, folhas de Manihot spp. e Enterolobium contortisiliquum. $\mathrm{O}$ destaque foi a intoxicação por casca de Coffea arabica em equinos, cuja ocorrência é rara, com 
relatos de intoxicação experimental em bovinos e equinos (DELFIOL et al., 2012).

Um número considerável de entrevistados mencionou a Asclepias curassavica como tóxica para bovinos e equinos, entretanto, a mesma é uma espécie de planta que foi comprovada como tóxica para bovinos, porém, não são ingeridas ou não são em quantidade suficiente para provocar intoxicação, portanto, planta sem interesse pecuário (TOKARNIA et al., 2012).

Quanto às outras plantas apontadas como tóxicas pelos entrevistados, necessita-se realizar futuros experimentos para comprovar sua toxicidade. No caso de Imperata brasiliensis Trin. e Cecropia hololeuca Miq. que foi mencionado hematúria em bovinos, deve-se realizar o diagnóstico diferencial para Pteridium arachnoideum, planta responsável pela maioria dos surtos deste trabalho.

As principais plantas tóxicas na região Sudoeste da Bahia que causam perdas para animais de fazenda são o Pteridium arachnoideum e Amorimia rigida.

Ressalta-se a importância da realização de estudos experimentais posteriores para Imperata brasiliensis Trin., Senna acuruensis, Capparis jacobina e Cecropia hololeuca Miq. A contribuição desta pesquisa abrange não só o meio científico, mas também acrescenta novos conhecimentos aos produtores rurais e técnicos da área.

\section{Agradecimentos}

Aos Professores Dr. Luciano Paganucci de Queiroz, Dr. ${ }^{\text {a }}$ Efigênia de Melo e Dr. Flávio França da Universidade Estadual de Feira de Santana (UEFS) pela identificação das espécies vegetais coletadas e ao INCT para o controle das intoxicações por plantas/CNPq (Proc. $\mathrm{n}^{\circ}$. 573534/2008-0) e CNPq (Proc. $\mathrm{n}^{\mathrm{o}}$. 475469/2012-7) pelo auxílio financeiro.

\section{Referências Bibliográficas}

1. ANJOS, B.L.; IRIGOYEN, L.F.; FIGHERA, R.A.; GOMES, A.D.; KOMMERS, G.D.; BARROS, C.S.L. Intoxicação aguda por samambaia (Pteridium aquilinum) em bovinos na Região Central do Rio Grande do Sul. Pesquisa Veterinária Brasileira, Seropédica, v.28, n.10, p.501-507, 2008.

2. ASSIS, T.S.; MEDEIROS, R.M.T.; ARAÚJO, J.A.S.; DANTAS, A.F.M.; RIET-CORREA, F. Intoxicações por plantas em ruminantes e equídeos no sertão paraibano. Pesquisa Veterinária. Brasileira, Seropédica, v.29, n.11, p.919924, 2009.

3. BEZERRA, C.W.C.; MEDEIROS, R.M.T.; RIVERO, B.R.; DANTAS, A.F.M.; AMARAL, F.R. Plantas tóxicas para ruminantes e equídeos da microrregião do Cariri Cearense. Ciência Rural, Santa Maria, v.42, n.6, p.10701076, 2012.

4. DANTAS, A.F.M.; RIET-CORREA F.; MEDEIROS, R.M.T.; GALIZA, G.J.N.; PIMENTEL, L.A.; ANJOS, B.L.; MOTA, T.A. Malformações congênitas em ruminantes no semiárido do Nordeste brasileiro. Pesquisa Veterinária 
Brasileira, Seropédica, v.30, n.10, p.807815, 2010.

5. DELFIOL, D.J.Z.; OLIVEIRA-FILHO, J.P.O.; $\quad$ CASALECCHI, $\quad$ F.L.; KIEVITSBOSCH, T.; HUSSNI, C.A.; RIETCORREA, F.; ARAÚJO JÚNIOR, J.P.; BORGES, A.S. Equine poisoning by coffee husk (Coffea arabica L.). BMC Veterinary Research, London, v.8, n.4, p.1-8, 2012.

6. FALBO, M.K.; REIS, A.C.F.; BALARIN, M.R.S.; BRACARENSE, A.P.F.R.L.; ARAÚJO, J.P.; OKANO, W.; SANDINI, I.E. Alterações hematológicas, bioquímicas, urinárias e histopatológicas na intoxicação natural em bovinos pela samambaia Pteridium aquilinum (L.) Kühn. Semina: Ciências Agrárias, Londrina, .26, n.4, p.547-558, 2005.

7. FURLAN, F.H.; MENDES, E.R.S.; DUCATTI， K.R.; MARCON， G.C.; DOMBROSKY, T.; AMORIM, T.M.; RIET-CORREA, F. Intoxicação aguda por Pteridium arachnoideum e Pteridium caudatum em bovinos e distribuição das plantas em Mato Grosso. Pesquisa Veterinária Brasileira, Seropédica, v.34, n.4, p.343-348, 2014a.

8. FURLAN, F.H.; COSTA, F.L.; TORRES, S.C.S.; KERBER, F.L.; DAMASCENO, E.S.; SALINO, A.; RIETCOOREA, F. Perfil de propriedades rurais com pastos invadidos por Pteridium arachnoideum na região norte de Mato Grosso e prevalência de hematúria enzoótica bovina. Pesquisa Veterinária Brasileira, Seropédica, v.34, n.8, p.753759, 2014b.

9. GABRIEL, A.L.; KOMMERS, G.D.; MASUDA, E.K.; FIGUERA, R.A.; PIAZER, J.V.M.; BARROS, C.S.L.; MARTINS, T.B.; ROSA, F.B. Aspectos clínico-hematológicos e lesões vesicais na intoxicação crônica espontânea por Pteridium aquilinum em bovinos. Pesquisa Veterinária Brasileira, Seropédica, v.29, n.7, p.515-525, 2009.
10. INSTITUTO BRASILEIRO DE GEOGRAFIA E ESTATÍSTICA. Pecuária 2013. Disponível em: <http://www.ibge.gov.br/estadosat/temas.p hp?sigla=ba\&tema=pecuaria2013>.

Acesso em: 05 jan. 2015.

11. KNUPP, S.N.R.; BORBUREMA, C.C.; OLIVEIRA NETO, T.S.; MEDEIROS, R.M.T.; KNUPP, L.S.; RIET-CORREA， F.; LUCENA， R.B. Surtos de fotossensibilização primária em equídeos causados por Froelichia humboldtiana. Pesquisa Veterinária Brasileira, Seropédica, v.34, n.12, p.11911195, 2014.

12. MEDEIROS, R.M.T.; BEZERRA, V.K.D.; RIET-CORREA, F. Intoxicação experimental por Froelichia humboldtiana em equinos. Ciência Rural, Santa Maria, v.44, n.10, p.1837-1840, 2014.

13. MELLO, G.W.S.; OLIVEIRA, D.M.; CARVALHO, C.J.S.; PIRES, L.V.; COSTA, F.A.L.; RIET-CORREA, F.; SILVA, S.M.M. Plantas tóxicas para ruminantes e equídeos no Norte Piauiense. Pesquisa Veterinária Brasileira, Seropédica, v.30, n.1, p.1-9, 2010.

14. OLIVEIRA, R.S.; SILVA, R.M.M.; DUTRA, P.A.; FERREIRA, E.A.; PINHEIRO, E.E.G.; MACÊDO, J.T.S.A.; PEDROSO, P.M.O. Intoxicação espontânea por Brachiaria decumbens em ovinos no estado da Bahia. Arquivo de Pesquisa Animal, Cruz das Almas, v.1, p.58-63, 2012.

15. PESSOA, C.R.M.; MEDEIROS, R.M.T.; RIET-CORREA, F. Importância econômica, epidemiologia e controle das intoxicações por plantas no Brasil. Pesquisa Veterinária Brasileira, Seropédica, v.33, n.6, p.752-758, 2013. 
16. PIMENTEL, L.A.; RIET-CORREA, F.; GUEDES, K.M.R.; MACÊDO, J.T.S.A.; MEDEIROS, R.M.T.; DANTAS, A.F.M. Fotossensibilização primária em equídeos e ruminantes no semi-árido causada por Froelichia humboldtiana (Amaranthaceae). Pesquisa Veterinária Brasileira, Seropédica, v.27, n.1, p.23-28, 2007.

17. REIS, S.D.S.; MACÊDO, J.T.S.A.; OLIVEIRA, R.S.; PEIXOTO, T.C.; FERREIRA, M.M.; GASPER, A.L.; PEDROSO, P.M.O. Enzootic hematuria in cattle from Northeastern Brazil. Brazilian Journal of Veterinary Pathology, Botucatu, v. 9, n.2, p.78-82, 2016a.

18. REIS, S.D.S.; OLIVEIRA, R.S.; MARCELINO, S.A.C.; MACÊDO, J.T.S.A.; RIET-CORREA, F.; PIMENTEL, L.A.; PEDROSO, P.M.O. Congenital malformations and other reproductive losses in goats due to poisoning by Poincianella pyramidalis (Tul.) L.P. Queiroz (=Caesalpinia pyramidalis Tul.). Toxicon, Oxford, 118:91-94, 2016b.

19. RIET-CORREA, F.; BEZERRA, C.W.C.; MEDEIROS, R.M.T. Plantas Tóxicas do Nordeste. Santa Maria: Sociedade Vicente Pallotti, 2011. 79p.

20. RISSI, D.R.; RECH, R.R.; PIEREZAN, F.; GABRIEL, A.L.; TROST, M.A.; BRUM, J.S.; KOMMERS, G.D.; BARROS, C.S.L. Intoxicações por plantas e micotoxinas associadas a plantas em bovinos no Rio Grande do Sul: 461 casos. Pesquisa Veterinária Brasileira, Seropédica, v.27, n.7, p.261-268, 2007.

21. ROTTA, E.; BELTRAME, L.C.C.; ZONTA, M. Manual de Prática de Coleta e Herborização de Material Botânico. Colombo: Embrapa, 2008. 31p.
22. SANTOS, J.R.S.; DANTAS, A.F.M.; RIET-CORREA, F. Malformações, abortos e mortalidade embrionária em ovinos causada pela ingestão de Mimosa tenuiflora (Leguminosae). Pesquisa Veterinária Brasileira, Seropédica, v.32, n.11, p.1103-1106, 2012.

23. SILVA, D.M.; RIET-CORREA, F.; MEDEIROS, R.M.T.; OLIVEIRA, O.F. Plantas tóxicas para ruminantes e equídeos no Seridó Ocidental e Oriental do Rio Grande do Norte. Pesquisa Veterinária Brasileira, Seropédica, v.26, n.4, p.223236, 2006.

24. SILVA, M.A.; SCÁRDUA, C.M.; DÓREA, M.D.; NUNES, L.C.; MARTINS, I.V.F.; DONATELE, D.M. Prevalência de hematúria enzoótica bovina em rebanhos leiteiros na microrregião do Caparaó, Sul do Espírito Santo, entre 2007 e 2008. Ciência Rural, Santa Maria, v.39, n.6, p.1847-1850, 2009.

25. SOUTO, M.A.M. KOMMERS, G.D.; BARROS, C.S.L.; PIAZER, J.V.M.; RECH, R.R.; RIET-CORREA, F.; SCHILD, A.L. Neoplasias do trato alimentar superior de bovinos associadas ao consumo espontâneo de samambaia (Pteridium aquilinum). Pesquisa Veterinária Brasileira, Seropédica, v.26, n.2, p.112-122, 2006.

26. SOUZA, P.E.C.; OILIVEIRA, S.S.; AGUIAR-FILHO, C.R.; CUNHA, A.L.; ALBUQUERQUE, R.F.; EVÊNCIONETO, J.; RIET-CORREA, F.; MENDONÇA, F.S. Primary photosensibilization in cattle caused by Froelichia humboldtiana. Research in Veterinary Science, v.93, n.3, p.13371340, 2012. 
27. TOKARNIA, C.H.; BRITO, M.F.; BARBOSA, J.D.; PEIXOTO, P.V.; DÖBEREINER, J. Plantas tóxicas do Brasil para Animais de Produção, 2.ed. Rio de Janeiro: Editora Helianthus, 2012. 566 .

28. VASCONCELOS, J.S.; RIETCORREA, F.; DANTAS, A.F.M.; MEDEIROS, R.M.T.; GALIZA, G.J.N.; OLIVEIRA, D.M.; PESSOA, A.F.A. Intoxicação por Mascagnia rigida (Malpighiaceae) em ovinos e caprinos. Pesquisa Veterinária Brasileira, Seropédica, v.28, n.10, p.521-526, 2008. 